\title{
Successful Treatment of a Late Diagnosed Esophageal Perforation with Mediastinitis and Pericardial Abscess
}

\author{
Mustafa Kupeli1 and Abdullah Dogan²
}

\begin{abstract}
We herein report a case of late diagnosed thoracic esophageal perforation. A 60-year-old man was diagnosed as an esophageal perforation with mediastinitis and pericardial abscess by detailed history taking, chest computed tomography, esophagoscopy and inflammatory findings in his blood test. Surgical drainage of mediastinum and pericardium was able to effectively control infectious process and pericardial fibrosis. Endoscopic esophageal stent placement on the thoracicesophageal fistula promoted healing of the esophageal wall defect and enabled him to restart oral intake. This case report suggests that detailed history taking is important for all patients. Effective drainage of mediastinum and the use of esophageal stent may be the treatment options for late-diagnosed esophageal perforation.
\end{abstract}

Key Words: Esophageal fistula, Mediastinitis, Pericardial fluid, Thoracotomy.

\section{INTRODUCTION}

Purulent pericarditis is seen rarely nowadays due to the development of effective antibiotic therapy. The most common cause of mediastinitis is perforation of the esophagus. Esophageal perforations result from foreign bodies, iatrogenic, spontaneously or esophageal tumors. Purulent pericarditis and mediastinitis due to esophageal perforation are associated with high morbidity and mortality since length of the hospital stay is long and the treatment is difficult. The morbidity and mortality in esophageal perforation is most often due to leakage of gastric content and food into the mediastinum. The therapeutic strategy may be conservative, or invasive. Surgical intervention is needed in the majority of incidents. ${ }^{1-3}$

We report a case of late-diagnosed esophageal rupture with purulent pericarditis and mediastinitis, that was successfully managed.

\section{CASE REPORT}

A 60-year-old previously healthy man felt as if food was stuck in his chest when he had dinner, but the symptom disappeared shortly thereafter. After a few days, the patient was admitted to cardiology clinic with chest pain. Transthoracic echocardiography of the patient revealed $1.7 \mathrm{~cm}$ localised pericardial effusion without cardiac tamponade. After 3-day treatment, he was discharged due to the recovery of chest pain and laboratory test parameters. The patient was admitted to this emergency department complaining of persistent fever, chest pain,

Department of Thoracic Surgeryl / Cardiovascular2, Gaziosmanpasa University, Tokat, Turkey

Correspondence: Dr. Mustafa Kupeli, Department of Thoracic

Surgery, Gaziosmanpasa University, Tokat, Turkey

E-mail: mustafakupeli@yahoo.com

Received: January 11, 2018; Accepted: August 07, 2018 shortness of breath 5 days after discharge. His complete blood count showed inflammatory findings: CRP, in $104.1 \mathrm{mg} / \mathrm{dl}$ and white blood cell count $22,190 / \mathrm{mm}^{3}$. His chest computed tomography (CT) demonstrated an increase in the size of the pericardial effusion, air in the pericardial sac and mediastinum but no mass lesion in esophagus (Figure 1). Under general anesthesia, fiberoptic and rigid esophagoscopy were performed. The esophageal perforation and purulent material coming from the perforation were seen in posterior part of the esophagus at 25th $\mathrm{cm}$ from the incisor teeth but no foreign body or esophageal mass were seen. A right thoracotomy was performed. Sero-purulent pleural effusion was aspirated and sent for biochemical analysis. Mediastinum and pericardium were very tense

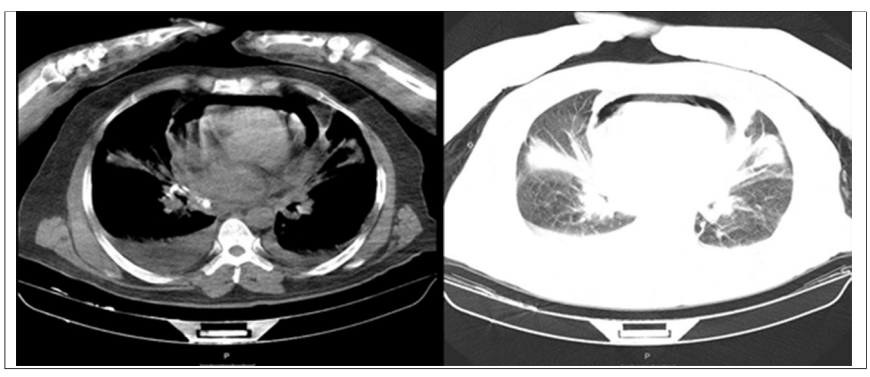

Figure 1: Thorax CT of patient.

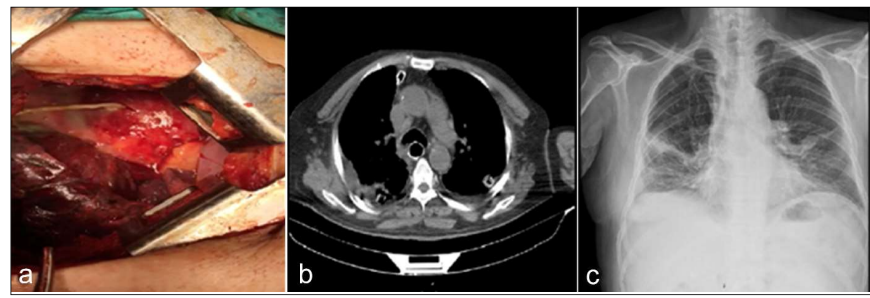

Figure 2: (a) Drainage of purulent material with right thoracotomy in pericardium intraoperatively.

(b) CT of patient with chest tubes and esophageal stent. (1 chest tube in right hemithorax, 1 chest tube in pericardium, 1 chest tube in left hemithorax and esophageal stent in esophagus).

(c) Control chest radiography of patient after 3 months. 
and hard. Mediastinum and pericardium were incised, and purulent material and air were drained (Figure 2a).

Pericardial dense fibrotic tissue was debrided. Pericardial window was opened. Two chest tubes were inserted in the thorax. A left anterior thoracotomy was performed in fifth midclavicular area to examine the left thoracic cavity and pericardium. Serous-purulent pleural fluid was drained. Pericardium was opened, and dense fibrotic tissue was debrided in the pericardium. A chest tube was inserted. An esophageal stent (ES) was placed for esophageal perforation (Figure $2 b$ ). The patient was referred to intensive care unit. Oral intake was stopped, parenteral nutrition was continued. ES migration was seen on postoperative day 22 and replaced. The patient was allowed to drink sips of water at 28 days postoperatively. On postoperative day 30 , he began a normal diet. He was discharged without complication on postoperative day 35 . The ES was removed successfully on postoperative day 71. The control porter-anterior (PA) chest $X$-ray was good and the patient had no complaint (Figure 2c).

\section{DISCUSSION}

Various etiologies, such as foreign bodies, tumors, iatrogenic or spontaneous tears can cause intrathoracic esophageal perforation. Intrathoracic esophagus and pericardium are located in the posterior and middle mediastinum, respectively, and the esophagus is attached to the parietal pericardium. 4 If the esophageal content contaminates the mediastinum due to esophageal tear, it causes inflammatory reaction and infection in the mediastinum and pericardium. Mediastinal infection and abscess can lead to serious clinical conditions such as sepsis and multiorgan failure which can cause death, if not treated immediately. ${ }^{3}$ In this case, we thought that rupture was caused by stuck food in the esophasus. The patient had not previously mentioned the history of food impaction until he was asked. Detailed history taking, and CT findings had alerted to think of esophageal tear and so esophagoscopy was performed. Detailed history taking is important in any case, especially in an older patient. 5

Esophageal perforation with mediastinitis is a lifethreatening disease. A delay in diagnosis may result in poor prognosis or mortality. Surgical or nonsurgical treatment option has been offered but there is no standard treatment procedure. Treatment is planned according to the elapsed time between perforation and diagnosis, size and location of the perforation, and the patient's health condition. Murakawa et al. suggested that the gold standard therapy is thoracotomy, laparotomy or both. They also suggested primary closure and esophagectomy according to the occurrence of sepsis. ${ }^{6}$ Although, these surgeries are also done with thoracoscopic and laparoscopic techniques nowadays, these techniques are performed for vitally stable patients and indications are limited. 6 Some authors suggest conservative treatment for esophageal perforation such as treatment with antibiotics, nilorally and ES. ${ }^{7}$ Latediagnosed esophageal fistula cannot be treated by primary suturing due to contaminated tissues. 8 In this case, the perforation was treated conservatively by using self-expandable covered ES, antibiotics and nil orally, since it was a delayed diagnosed case so the option of repairing the tear with sutures due to necrosis, inflammation and contaminated environment was not considered.

Mediastinitis must be treated in esophageal perforation. Draining the purulent material in mediastinum is the main goal in the treatment of mediastinitis. The appropriate drainage is performed by surgical intervention. ${ }^{2}$ Surgical intervention can be done with thoracoscopic or open surgery. In this patient, it was tried to perform thoracoscopic surgery but after noting the mediastinum and pericardium to be very thuk and regid, it was decided to perform open surgery. It was a wise decision that the open surgery was performed since there were fibrotic tissues in the pericardium and it could not be debrided by thoracoscopic technique.

In conclusion, detailed history taking is important in older patients, especially who have chest pain. Draining purulent material and debridement of necrotic and fibrotic tissues in mediastinal space and pericardium are important and should be the main goal of the treatment of esophageal perforation; and ES can be used to control the contamination.

\section{REFERENCES}

1. Petcu CP, Dilof R, Bataiosu C, Petcu PD. Purulent pericardial effusions with pericardial tamponade - diagnosis and treatment issues. Curr Health Sci J 2013; 39:53-6.

2. Schizas NC, Paliouras D, Rallis T, Gogakos AS, Lazopoulos A, Chatzinikolaou F, et al. Successful surgical treatment of a complicated esophageal perforation, by use of primary closure and lung parenchyma reinforcement. Ann Transl Med 2016; 4:448.

3. Kimura T, Takemoto T, Fujiwara Y, Yane K, Shiono H. Esophageal perforation caused by a fish bone treated with surgically indwelling drainage and fibrin glue injection for fistula formation. Ann Thorac Cardiovasc Surg 2013; 19:289-92.

4. Marar D, Muthusamy V, Krishnan SC. Avoiding oesophageal injury during cardiac ablation: insights gained from mediastinal anatomy. Europace 2018; 20:466-71.

5. Ghosh D, Karunaratne P. The importance of good history taking: a case report. J Med Case Rep 2015; 9:97.

6. Murakawa K, Ono K, Yamamura Y, Niwa H, Yamamoto H, Muto J, et al. Minimally invasive surgery for esophageal cancer after esophageal perforation. Asian J Endosc Surg 2017; 10: 407-10.

7. Aronberg RM, Punekar SR, Adam SI, Judson BL, Mehra S, Yarbrough WG. Esophageal perforation caused by edible foreign bodies: a systematic review of the literature. Laryngoscope 2015; 125:371-8.

8. Kircheva DY, Vigneswaran WT. Successful primary repair of late diagnosed spontaneous esophageal rupture: A case report. Int J Surg Case Rep 2017; 35:49-52.

$$
\text { ................. }
$$

